\title{
Raman Scattering from a Nematic Liquid Crystal: Orientational Statistics
}

\section{Citation}

Jen, Shen, Noel A. Clark, Peter S. Pershan, and E. B. Priestley. 1973. Raman scattering from a nematic liquid crystal: Orientational statistics. Physical Review Letters 31(26): 1552-1556.

\section{Published Version}

doi:10.1103/PhysRevLett.31.1552

\section{Permanent link}

http://nrs.harvard.edu/urn-3:HUL.InstRepos:10361962

\section{Terms of Use}

This article was downloaded from Harvard University's DASH repository, and is made available under the terms and conditions applicable to Other Posted Material, as set forth at http:// nrs.harvard.edu/urn-3:HUL.InstRepos:dash.current.terms-of-use\#LAA

\section{Share Your Story}

The Harvard community has made this article openly available.

Please share how this access benefits you. Submit a story.

Accessibility 
applied to the $x$ axis, and the output of the acoustic (or sideband) wave was applied to the $y$ axis of an $x-y$ recorder. Beam current was then changed to sweep out the curve. Curves obtained this way are somewhat erratic because of temporal fluctuations in all amplitudes; the curves shown represent averages of these fluctuations. Threshold occurs for a beam current of approximately $500 \mu \mathrm{A}$, but depends also on beam energy and plasma density.

The spatial behaviors of the amplitudes of the three waves, for three values of beam current, are shown in Figs. 4(b)-4(d). The wave amplitudes were measured by tuning a receiver in the manner described above. As the beam current is increased, the beam wave amplitude is observed to grow more rapidly and to saturate nearer to the gun. The maximum amplitudes of the acoustic and Trivelpiece-Gould modes likewise tend to shift towards the gun.

Well above threshold, significant broadening of the sideband and acoustic spectra takes place, while the peak amplitude of the sideband modes grows to a level as high as $30 \%$ of the beam mode. This suggests that the energy content of the sideband modes can be comparable to that of the beam mode, which then implies that saturation of the beam mode may be associated with pump deple- tion. ${ }^{7}$ Clearly, further experiments and a more complete theoretical model will be required in order to establish that this nonlinear mechanism is indeed strong enough to limit the amplitude of the beam instability.

The authors wish to acknowledge valuable discussions with Professor A. Bers and Professor B. Coppi.

*Work supported by the National Science Foundation under Grant No. GK-28282X1.

${ }^{1}$ W. Drummond et al., Phys. Fluids 13, 2422 (1970); T. O'Neil, J. Winfrey, and J. Malmberg, Phys. Fluids 14, 1204 (1971); T. O'Neil and J. Winfrey, Phys. Fluids $\underline{15}, 1514$ (1972).

${ }^{2}$ K. Nishikawa, J. Phys. Soc. Jap. 24, 916 (1968).

${ }^{3}$ R. Stenzel and A. Wong, Phys. Rev. Lett. 28,274 (1972).

${ }^{4}$ M. Porkolab, V. Arunasalam, and R. Ellis, Jr., Phys. Rev. Lett. 29, 1438 (1972)

${ }^{5}$ A. L. Throop and R. R. Parker, Research Laboratory of Electronics, Massachusetts Institute of Technology Quarterly Progress Report No. 108, 1973 (unpublished), pp. 154-167.

${ }^{6}$ B. Coppi, M. Rosenbluth, and R. Sudan, Ann. Phys. (New York) 55, 207 (1969); M. Rosenbluth, B. Coppi, and R. Sudan, Ann. Phys. (New York) 55, 248 (1969).

${ }^{7}$ D. DuBois and M. Goldman, Phys. Rev. 164, 207 (1967) .

\title{
Raman Scattering from a Nematic Liquid Crystal: Orientational Statistics*
}

\author{
Shen Jen, Noel A. Clark, and P. S. Pershan \\ Division of Engineering and Applied Physics, Harvard University, Cambridge, Massachusetts 02138
}

and

E. B. Priestley

RCA Laboratories, Princeton, New Jersey 08540

(Received 28 September 1973)

\begin{abstract}
Raman-scattering techniques have been used to obtain a new quantitative measure of orientational statistics of individual molecules in a nematic liquid crystal. A marked discrepancy is observed between these measurements and the predictions of existing theories of nematic ordering.
\end{abstract}

Statistical theories of nematic liquid crystals usually begin with the assumption that the molecules are rigid and cylindrical. ${ }^{1}$ Taking $\cos \theta$ to be the direction cosine between the principal axis of an individual molecule and the nematic axis, theories have been developed to predict the probability distribution $f(\cos \theta)$ for individual molecules $\left[\int_{-1}^{1} f(\cos \theta) d \cos \theta=1\right] .^{2-6}$ All previous ex- perimental efforts to characterize the nematic order consist of measurements of the anisotropy in tensors of the second rank which, under the assumptions mentioned above, are equivalent to measurements of $S=\left\langle P_{2}(\cos \theta)\right\rangle=\int_{-1}{ }^{1} P_{2}(x) f(x) d x$, where $P_{2}(x)$ is the Legendre polynomial of order $2 .^{3,7^{-11}}$ We present here the first simultaneous measurements of both $\left\langle P_{2}(\cos \theta)\right\rangle$ and $\left\langle P_{4}(\cos \theta)\right\rangle$ 
in any nematic liquid crystal and demonstrate disagreement between the experimental results and existing theories. We argue that in view of the number of adjustable parameters, the excellent agreement that has been reported between recent self-consistent mean field theories and measured values of $\left\langle P_{2}(\cos \theta)\right\rangle^{3}$ is not a meaningful test of the theories or of the assumption of rigid rodlike molecules.

We have used a Raman-scattering technique ${ }^{12}$ to measure both $\left\langle P_{2}\right\rangle$ and $\left\langle P_{4}\right\rangle$ for a probe molecule, $\mathrm{N}$-( $p^{\prime}$-butoxybenzylidene)- $p$-cyanoaniline (BBCA), dissolved in $\mathrm{N}-p^{\prime}$-methoxybenzylidene)$p$-n-butylaniline (MBBA). We will present evidence that the values for $\mathrm{BBCA}$ are also characteristic of the MBBA host. The bond axis of the $\mathrm{C} \equiv \mathrm{N}$ group at the end of the BBCA molecule is nearly parallel to the major axis of the molecule and there is a strong, narrow, anisotropic Raman line associated with the $\mathrm{C} \equiv \mathrm{N}$ stretching vibration that can be spectroscopically isolated from all of the other Raman lines of the solution. The Raman polarizability tensor for the localized $\mathrm{C} \equiv \mathrm{N}$ vibration has the uniaxial form $\overrightarrow{\boldsymbol{\alpha}}_{\mathrm{CN}}$ $=a \delta_{i j}+(b-a) \delta_{i 3} \delta_{j 3}$, where the third direction coincides with the uniaxial direction of the $\mathrm{C} \equiv \mathrm{N}$ bond. Since $a \neq b$, the Raman cross section for polarized light scattered by a BBCA molecule is dependent on the orientation of the molecule relative to polarization directions. We denote the integrated intensity per unit solid angle for scattered light polarized along $z$ with incident light polarized along $x$ as $I_{x z}$. Since there is no phase coherence between $\mathrm{C} \equiv \mathrm{N}$ vibrations on different molecules, the quantity $I_{x z}$ is a statistical average for individual BBCA molecules. Thus in the nematic phase (take the nematic axis along $z$ ) the depolarization ratios $\rho_{1} \equiv \boldsymbol{I}_{z x} / \boldsymbol{I}_{z z}$ and $\rho_{2} \equiv I_{x z} / I_{x x}$ contain information on the statistical averages $\left\langle P_{2}(\cos \theta)\right\rangle$ and $\left\langle P_{4}(\cos \theta)\right\rangle$ for a $\mathrm{BBCA}$ molecule. In the isotropic phase of BBCA-MBBA solutions at different concentrations, measurements of $\rho_{1}$ $=\rho_{2}$ determine the ratio $a / b=0.06 \pm 0.02$ to be independent of temperature and concentration. $\mathrm{Ra}-$ man spectra in the nematic phase were observed using monodomain, planar samples, with thicknesses varying from 25 to $250 \mu \mathrm{m}$, homogeneous ly aligned between glass slides. Several alignment techniques (e.g., rubbing) gave identical results. ${ }^{13}$ The back-scattering geometry was used with incident light ( $\lambda=5145$ or $6471 \AA$ ) normal to the sample $x, z$ plane. In order to extract $\left\langle P_{2}\right\rangle$ and $\left\langle\boldsymbol{P}_{4}\right\rangle$ from the measured values of $\rho_{1}$ and $\rho_{2}$, several factors must be taken into account. In samples of finite thickness multiple scattering by the direction fluctuations causes spurious depolarization of incident and scattered light. This effect was eliminated by measuring $\rho_{1}$ and $\rho_{2}$ as a function of thickness and extrapolating to zero thickness. Secondly, the measured ratios must be corrected for the differences in solid angle outside and inside the sample, anisotropic demagnification of the scattering volume, and anisotropic reflection effects at the liquid-crystal-glass interface. ${ }^{14}$ Finally, a small correction must be made for the fact that the $\mathrm{C} \equiv \mathrm{N}$ bond axis is not colinear with the long axis of $\mathrm{BBCA}_{\text {, there being }}$ an angle $\beta$ between them which we estimate to be $10^{\circ} \pm 2^{\circ}$. Using $a / b, \rho_{1}$, and $\rho_{2}$ with these corrections, one can obtain $\left\langle\cos \theta^{2,4} \theta\right\rangle$ and, hence, $\left\langle P_{2,4}(\cos \theta)\right\rangle$ for $\mathrm{BBCA}$ in MBBA. These results are shown in Fig. 1.

Data were taken at $10 \%$ and $20 \%$ mole fraction of BBCA in MBBA. Although the temperature for the isotropic to nematic phase transition $\left(T_{c}\right)$ changes with the BBCA concentration, the results are independent of concentration when plotted as a function of $\boldsymbol{T}_{c}-T$. In order to confirm that the statistics of the probe molecule are also characteristic of MBBA, we compare, in Fig. 1, our Raman measurements of $\left\langle P_{2}\right\rangle$ for the BBCA probe with five independent measurements of $\left\langle P_{2}\right\rangle$ for MBBA. These are absolute NMR measurements of partially deuterated MBBA, ${ }^{15}$ measurements of the optical ${ }^{16,17}(\Delta \alpha)$ anisotropy, and three separate measurements of the magnetic ${ }^{9-11}(\Delta \chi)$ anisotropy of MBBA. Relative values of $\left\langle P_{2}\right\rangle$ from the latter measurements were obtained by assuming $\left\langle P_{2}\right\rangle$ is proportional to $\Delta \alpha$ and $\Delta \chi$ and fixing the proportionality factor to obtain agreement at $T_{c}-T=2^{\circ} \mathrm{C}$. The temperature dependence of $\left\langle P_{2}\right\rangle$ calculated from the different measured anisotropies of MBBA are identical to the temperature dependence obtained from the Raman data on the BBCA molecule. This result, along with the structural similarity of BBCA and MBBA, supports our contention that the orientational statistics of the BBCA guest are similar to those of the MBBA host.

The results of the Maier-Saupe theory (MS) for $\left\langle P_{2}\right\rangle$ and $\left\langle P_{4}\right\rangle$ are also included in Fig. $1 .^{2} \mathrm{Al}-$ though they do show the qualitative behavior of the measured quantities, there are significant quantitative discrepancies. Extensions of the MS theory, including molecular field terms varying as $P_{4}(\cos \theta)$, have been proposed. ${ }^{3,4}$ These results are also shown in Fig. 1 for the choice of parameters ${ }^{3}[\lambda=-0.55$ and $\gamma=10]$ that obtain the best 


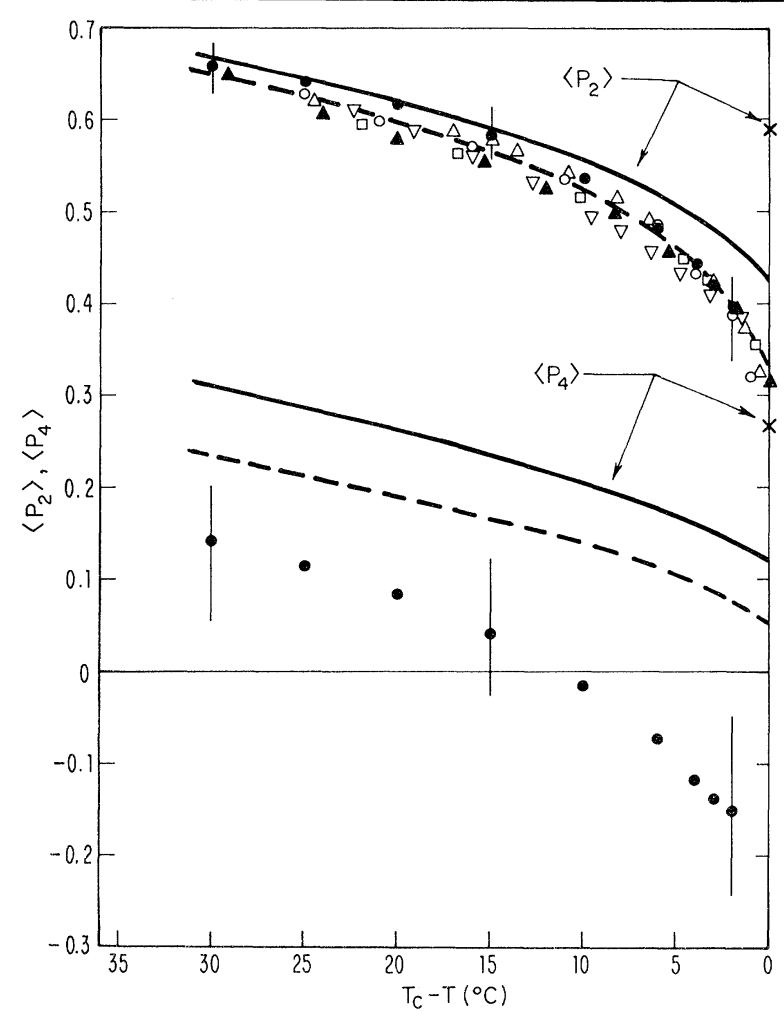

FIG. 1. Theoretical and experimental values of the nematic order parameters $\left\langle P_{2}\right\rangle$ and $\left\langle P_{4}(\cos \theta)\right\rangle$ [solid line, theoretical results of MS (Ref. 2); dashed line, HJL (Ref. 3) with $\lambda=-0.55$ and $\gamma=10$; crosses, Onsager-Lakatos (Ref. 6); filled circles, present Raman measurements; open circles, NMR results on partially deuterated MBBA (Ref. 15); squares, relative values obtained from measurements of the optical anisotropy $\Delta \alpha$ (Ref. 17)] and of the magnetic anisotropy $\Delta x$ (inverted triangles, Ref. 11; open triangles, Ref. 10; filled triangles, Ref. 9). The error bars on the present Raman measurements reflect uncertainties in the value of $\beta$ as well as experimental uncertainties.

fit to the experimental values of $\left\langle P_{2}\right\rangle$. Although with the extra free parameters of the HumphriesJames-Luckhurst ${ }^{3}$ (HJL) theory, the fit to the $\left\langle P_{2}\right\rangle$ data is perfect, the fit to the $\left\langle P_{4}\right\rangle$ data is not significantly improved. Furthermore, the choice of $\gamma=10 \pm 2$ that is required to fit the $\left\langle P_{2}\right\rangle$ data implies an unusually strong dependence of order parameter on density. With more reasonable values of $\gamma$ [i.e., $\gamma=4$ ], the fit of the HJL theory to $\left\langle P_{2}\right\rangle$, although no longer perfect, improves upon the MS theory. The predictions for $\left\langle P_{4}\right\rangle$, however, still disagree significantly with the data. Thus the most important discrepancy between theory and experiment concerns the values of $\left\langle P_{4}\right\rangle$. To examine this more carefully, consider the first three terms in the general expansion of $f(\cos \theta)$ in Legendre polynomials:

$$
f^{I I I}(\cos \theta)=\frac{1}{2}\left[1+5\left\langle P_{2}\right\rangle P_{2}(\cos \theta)+9\left\langle P_{4}\right\rangle P_{4}(\cos \theta)\right] .
$$

In Fig. 2 we plot $f^{111}$ for two temperatures using experimental values of $\left\langle P_{2}\right\rangle$ and $\left\langle P_{4}\right\rangle$ and values calculated from the HJL model after the parameters were adjusted to obtain good agreement with $\left\langle P_{2}\right\rangle$. Note that although $f(\cos \theta)$ is positive definite $f^{I I I}(\cos \theta)$ need not be. The principal result to see in Fig. 2 is that the BBCA (and presumably also the MBBA) molecules have a stronger tendency to be tipped away from the nematic axis than predicted by mean field theory. This tendency is strongest near the nematic-isotropic phase transition. Aside from the mean field theories, other statistical mechanical models of nematic ordering are available. Specifically there is the work of Onsager ${ }^{5}$ for the ordering of a system of hard rods as a function of density. Using the Onsager model, Lakatos ${ }^{6}$ has recently calculated values of $\left\langle P_{n}(\cos \theta)\right\rangle$ for all $n$. In Fig. 1 we also show these results for $\left\langle P_{2}\right\rangle$ and $\left\langle P_{4}\right\rangle$ at the transition density. The ratio of $\left\langle P_{4}\right\rangle /\left\langle P_{2}\right\rangle$ as a function of $\left\langle P_{2}\right\rangle$ is basically the same as the results of mean field theory and also disagrees with our results. We now speculate as to possible reasons for these differences.

It was $^{1 \text { a }}$ pointed out that for molecules which are not cylindrically symmetric the angular distribution function $f(\cos \theta)$ must be replaced by a more general function of two Eulerian angles. In view of the fact that the $\mathrm{C} \equiv \mathrm{N}$ axis is not precisely parallel to the major axis of the BBCA molecule, neglect of this effect could, in principle, lower the value of $\left\langle P_{4}\right\rangle$ deduced from the measured values of $\rho_{1}$ and $\rho_{2}$. However, we have found in practice that the value of $\left\langle P_{4}\right\rangle$ obtained from the Raman data is negligibly changed even when very large anisotropies of the Alben type are included, a result of the near parallelism of the $\mathrm{CN}$ bond and the principal molecular axis.

On the other hand, mean field theory assumed rigid molecules, an assumption that is clearly questionable. $^{1 \mathrm{~b}_{0} 1 \mathrm{c}}$ The butyl tail of MBBA is quite flexible and we have no knowledge of the statistical distribution of different conformers in MBBA. To the extent molecules are not rigid there is no unique choice for a nematic order parameter. In the present case the agreement between the NMR results and all the other measure- 


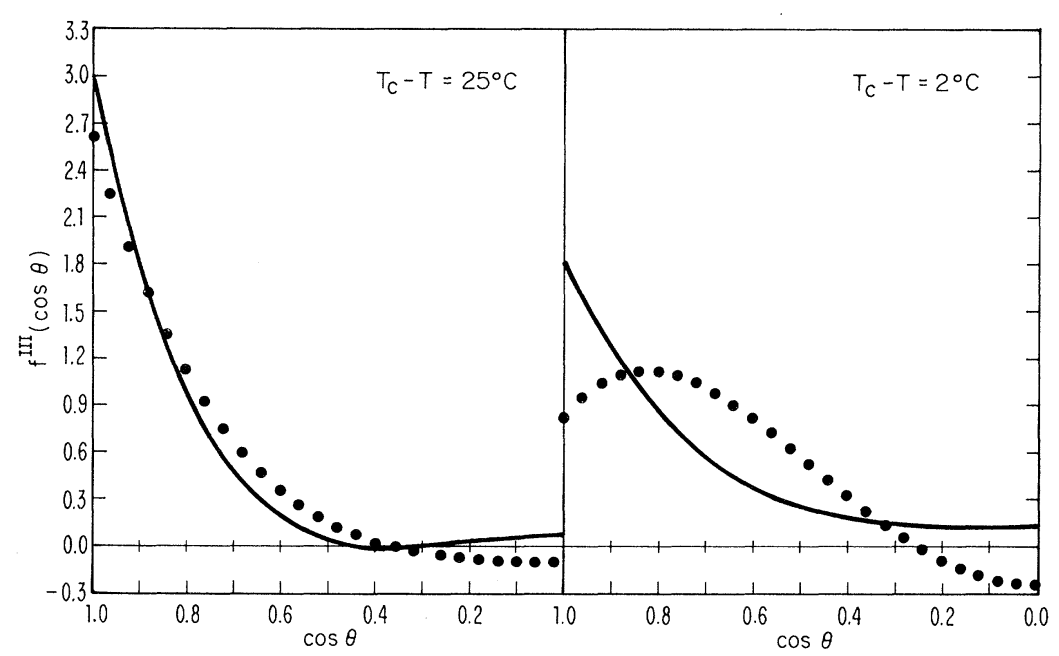

FIG. 2. Plot of the theoretical and experimental truncated angular distribution functions $f^{\text {III }}(\cos \theta)$ : solid line, HJL (Ref. 3); filled circles, Raman measurements.

ments of $\left\langle P_{2}\right\rangle$ suggest that $\left\langle P_{2}(\cos \theta)\right\rangle$ may be suitable if $\cos \theta$ is the direction $\operatorname{cosine}$ of the rigid part of MBBA with respect to the nematic axis. In that case there is no a priori reason to expect that the mean field theory should apply to only one part of a complex molecule. For example, if $L$-shaped isomers were relatively probable, the observed behavior of $\left\langle P_{4}\right\rangle$ could be rationalized. Of course it is also possible that the approximation of a rigid molecule is acceptable, but that the mean field theory fails because it neglects interactions that induce splay on a microscopic scale, i.e., pair correlations in which neighboring molecular axes are not parallel. For example, dipole-dipole interactions could do this.

In summary, although existing statistical theories of nematic ordering are qualitatively useful for understanding the gross features of nematic ordering, they are not quantitatively reliable.

We would like to express our appreciation to Professor Robert Meyer, Professor David Dolphin, and Dr. Peter Wojtowicz for conversations and other guidance during the course of this work. In addition we thank Dr. Yat-Shir Lee for making his unpublished data available to us.

*Work supported in part by the Joint Services Electronics Program (U.S. Army, U.S. Navy, and U.S. Air Force) under Contract No. N00014-67-A-0298-0006 and by the National Science Foundation under Grants No. GH-33576 and No. GH-34401.

${ }^{1}$ For exceptions to this see (a) R. Alben, J. R. Mc Coll, and C. S. Shih, Solid State Commun. 11, 1081 (1972); (b) F. Jähnig, to be published; (c) H. Stenschke, Solid
State Commun. 10, 653 (1972).

${ }^{2}$ W. Maier and A. Saupe, Z. Naturforsch. 13a, 564 (1958), and 14a, $882(1959)$, and 15a, $287(\overline{1960})$.

${ }^{3}$ R. L. Humphries, P. G. James, and G. R. Luckhurst, J. Chem. Soc., Faraday Trans. 2 68, 1031 (1972).

${ }^{4}$ S. Chandrasekhar and N. V. Madhusudana, Mol. Cryst. Liquid Cryst. 10, 151 (1970).

${ }^{5}$ L. Onsager, Ann. N.Y. Acad. Sci. 51, 627 (1949).

${ }^{6} \mathrm{~K}$. Lakatos, J. Status. Phys. 2, 121 (1970).

${ }^{7}$ A. Saupe and W. Maier, Z. Naturforsch. 16a, 816 (1971); P. Pincus, J. Phys. (Paris) 30, C4-8 (1969).

${ }^{8}$ N. V. Madhusudana, R. Shashidhar, and S. Chandrasekhar, Mol. Cryst. Liquid Cryst. 13, 61 (1971); S. Chandrasekhar and N. V. Madhusudana, J. Phys. (Paris) 30, C4-24 (1969).

${ }^{9}$ G. Sigaud and H. Gasparoux, J. Chim. Phys. Physicochim. Biol. 70, 699 (1973).

${ }^{10} \mathrm{I}$. Haller, J. Chem. Phys. 57, 1400 (1972).

${ }^{11} \mathrm{P}$. I. Rose, presented at the Fourth International Liquid Crystal Conference, Kent State University, Kent, Ohio, 1972 (to be published).

${ }^{12}$ E. B. Priestley, P. S. Pershan, R. B. Meyer, and D. H. Dolphin, Vijnana Parishad Anusandhan Patrika 14, 93 (1971); E. B. Priestley and P. S. Pershan, to be published.

${ }^{13}$ P. Chatelain, Bull. Soc. Fr. Mineral. Cristallogr. 66, 105 (1943); J. L. Janning, Appl. Phys. Lett. 21, 173 (1972); E. Guyon, P. Pieranski, and M. Boix, to be published.

${ }^{14}$ M. Lax and D. F. Nelson, in Coherence and Quantum optics, edited by L. Mandel and E. Wolf (Plenum, New York, 1973), p. 415.

${ }^{15}$ Y. S. Lee, Y. Y. Hsu, and D. Dolphin, presented at the American Chemical Society Symposium on Ordered Fluids and Liquid Crystals, Chicago, Mlinois, 1973 (to be published).

${ }^{16}$ Madhusudana, Shashidhar, and Chandrasekhar (Ref. 8) first drew attention to the empirical fact that the temperature dependence of the anisotropy in the optical- 
frequency polarizability tensor, $\Delta \alpha$, sćales with other measures of $\left\langle\boldsymbol{P}_{2}\right\rangle$. The proportionality between $\Delta \alpha$ and nematic order is direct with no need for local-field corrections. Similarly, the Raman data for $\left\langle\boldsymbol{P}_{2}\right\rangle$ are in excellent agreement with other data for $\left\langle P_{2}\right\rangle$ without introduction of local-field effects. The empirical fact that local-field corrections are not necessary for either of these measurements can be rationalized as follows: Local-field corrections depend on dipole-dipole summations over neighboring molecules. Although the problem can be solved in a number of ways, the answers are ultimately dependent on near-neighbor correlations. Near-neighbor correlations appear to be relatively insensitive to temperature implying the same about the local-field corrections. In the present case the ratio $a / b$ in the isotropic phase, which should also reflect local-field effects, was measured to be independent of temperature and we assume the same value applies to the nematic phase. The $\left\langle\boldsymbol{P}_{2}\right\rangle$ data support this assumption.

${ }^{17} \Delta \alpha$ was obtained from our refractive index measurements and the "Vuks equation" (see Ref. 8).

\title{
Comment on " $\pi$ Condensation in Nuclear Matter"*
}

\author{
R. F. Sawyer \\ Aspen Center for Physics, Aspen, Colorado 81611, and Department of Physics, \\ University of California, Santa Barbara, California 93106
}

(Received 14 September 1973)

\begin{abstract}
Recent remarks on the possibility of negative-pion condensation in superdense nuclear matter are shown to be based on an incorrect formalism. The possibilities of $\pi^{-}$and $\pi^{0}$ condensation are compared and some problems are enumerated which must be solved before it is known which, if either, of the phenomena can occur.
\end{abstract}

In an interesting recent Letter, mainly concerned with neutral-pion condensation in superdense nuclear matter, Migdal has made some remarks on the possibility of a charged-pion condensation. ' However, his treatment is based on a formal approach which is untenable for the charged-meson problem.

At the very beginning of Ref. 1 it is stated that the critical condition for $\pi^{-}$condensation in a neutron gas is given by the solution to the equation

$$
\overrightarrow{\mathrm{k}}^{2}+m_{\pi}^{2}-\mu^{2}+\Pi(\mu, \overrightarrow{\mathrm{k}})=0 .
$$

Here $\mu=\mu_{n}-\mu_{p}$ is the difference between the neutron and proton chemical potentials; $k$ is the wave vector of the $\pi^{-}$mode; $\Pi(\omega, \vec{k})$ is the proper polarization part for the $\pi$ - propagating in the neutron medium. Migdal further states that in the limit of a vanishing number of pions (and protons), just above the critical density, the parameter $\mu=\mu_{n}-\mu_{p}$ will be equal to the Fermi energy of the neutrons.

I shall demonstrate below that Eq. (1) can be used to derive the critical density when the correct value of $\mu$ is used. But the correct value of $\mu$ is so different from $\epsilon_{n}{ }^{\mathrm{F}}$ that Migdal's remarks based on this equation have no relevance to the $\pi^{-}$problem. The chemical potential difference $\mu_{n}-\mu_{p}$ must be derived dynamically from the formula for the condensation energy. Even in the case of infinitesimal pion density, $\mu$ will differ from $\epsilon_{n}{ }^{\mathrm{F}}$ by a finite and large amount. Furthermore, one can look directly at the energy of the system to see that the solution of (1), with $\epsilon$ $=\epsilon_{n}{ }^{\mathrm{F}}$, is unrelated to any phase transition.

Since the $\pi^{-}, \pi^{+}$condensation problem has been treated elsewhere in some detail ${ }^{2-4}$ for realistic values of the parameters, I discuss here a limit $M_{N} \rightarrow \infty$, in which the problem is trivially solvable and which illustrates the important points of principle.

The $\pi^{-}$polarization operator in a free Fermi gas of neutrons, calculated to second order in the pion-nucleon coupling constant $f$, comes directly from the crossed graph with an intermediate proton and is given by

$$
\Pi(\omega, k)=-2 f^{2} k^{2} \rho / \omega m_{\pi}^{2},
$$

where $\rho$ is the density of neutrons. If one looks at Eq. (1), takes $\mu=\epsilon_{\mathrm{F}} \rightarrow 0$ (as $M_{N} \rightarrow \infty$ ), and uses the expression for $\Pi$ given in $\mathrm{Eq}$. (2), it will be found that there will be a solution for arbitrarily small values of $f, k$, or $\rho$, in the limit $M_{N} \rightarrow \infty$. That is, in the static limit a $\pi^{-}$condensation is predicted for arbitrarily small coupling, or density.

To show that this is a defect of the formalism and not a pathology of the static limit, consider 